\title{
Semi-Regular 3D Mesh Progressive Compression and Transmission based on an Adaptive Wavelet Decomposition
}

\author{
Céline Roudet ${ }^{a, b}$, Florent Dupont ${ }^{a, b}$ and Atilla Baskurt ${ }^{a, c}$ \\ ${ }^{a}$ Université de Lyon, CNRS \\ ${ }^{b}$ Université Lyon 1, LIRIS, UMR5205, F-69622, France. \\ ${ }^{c}$ INSA-Lyon, LIRIS, UMR5205, F-69621, France.
}

\begin{abstract}
We introduce a new patch-based multi-resolution analysis of semi-regular mesh surfaces. This analysis brings patch-specific wavelet decomposition, quantization and encoding to the mesh compression process. Our underlying mesh partitioning relies on surface roughness (based on frequency magnitude variations), in order to produce patches, representative of semantic attributes of the object. With current compression methods based on wavelet decomposition, some regions of the mesh still have wavelet coefficients with a non negligible magnitude or polar angle (the angle with the normal vector), reflecting the high frequencies of the model. For each non-smooth region, our adaptive compression chain provides the possibility to choose the best prediction filter adjusted to its specificity. Our hierarchical analysis is based on a semi-regular mesh decomposition produced by second-generation wavelets. Apart from progressive compression, other types of applications can benefit from this adaptive decomposition, like error resilient compression, view-dependent reconstruction, indexation or watermarking. Selective refinement examples are given to illustrate the concept of ROI (Region Of Interest) decoding, which few people has considered, whereas it is possible with JPEG2000 for images.
\end{abstract}

Keywords: Adaptive compression, view-dependent reconstruction, ROI decoding, mesh segmentation, mesh classification, multiresolution analysis, geometric wavelet, lifting scheme.

\section{INTRODUCTION}

Three dimensional objects and scenes find their way into more and more applications, thanks to the ever increasing bandwidth available in modern telecommunication networks, such as the High Speed Internet, or $3 \mathrm{G}$ mobile networks. At the same time, user expectations for rendering require increasingly complex 3D models. A triangle mesh is actually the most common representation for objects and scenes. This modelling includes geometry and topology information, which can be expensive in terms of computation, storage, and transmission, even with today's devices and networks.

Multiresolution (MR) techniques for triangle meshes with arbitrary topology was introduced by Lounsbery, ${ }^{1}$ in order to represent data with multiple Levels of Detail (LOD). This scalable representation is commonly produced by a wavelet transform, which was extended for irregular sampled signals like meshes. Two approaches are possible, considering directly the irregular structure or using a subdivision scheme as a scaling function basis. In the latter case, the canonical quadrisection produced by the subdivision schemes imposes to apply an analysis on semi-regular (SR) meshes. These are considered as functions via the parameterizations inherently defined by the remeshing.

In this paper, we consider the SR surface representation because the target applications do not impose a lossless compression scheme. This paradigm delivers efficient data structures and processing algorithms, closer to the ones used for data sampled on regular grids.

The main SR remeshing algorithms rely on the same philosophy, reducing most of the connectivity and parametric information from the mesh. This way, details are represented only with geometric attributes. For that purpose, the mesh is simplified to produce the base complex on which the input model will be parameterized.

Corresponding author is currently at Université Lyon 1, Bâtiment NAUTIBUS, 8 boulevard Niels Bohr, F-69622 Villeurbanne Cedex - France. Email: croudet@liris.cnrs.fr, Telephone: +33 (0)4 2623 44 64, Fax: +33 (0)4 72431536 . 
Two approaches are considered: chartification or progressive decimations. Finally, the resampling stage may vary but is always based on subdivision connectivity construction.

The first inception was introduced by Eck et al. ${ }^{2}$ They partitioned the original mesh into Voronoï tiles. Its dual construction (the Delaunay triangulation) was used to produce the coarse model. However, it is possible to take more into account the geometric and visual properties of the initial surface, during the base complex construction. Gioia ${ }^{3}$ experimentally obtained on average twice less wavelet coefficients than with the previous method, for natural and CAD objects. They both used harmonic maps to construct the local parameterization, which minimizes the distortion when mapping a curved surface to the plane.

The other class of methods use progressive decimations based on constructing a mesh hierarchy thanks to the vertex removal or edge collapse operation. In the MAPS algorithm, ${ }^{4}$ the vertices chosen to be removed are those that withdraw the fewest geometrical and topological information. A conformal mapping, consisting in expressing the decimated vertices as barycentric coordinates, is used, together with the Loop subdivision to produce the SR mesh. But Guskov et al. observed that this decimating context is favorable for constructing a global parameterization. They used it together with a recursive piercing procedure and unlifted Butterfly wavelets in their Normal Mesh algorithm. ${ }^{5}$ The resulting meshes are ideally suited for progressive compression, because most of the geometric details are concentrated along the surface normal, expressed with a single scalar. This algorithm currently produces one of the best remeshes for compression, but only for closed surfaces.

Because most of the previously described algorithms suffered from smoothness artefacts at patch boundaries, a new class of algorithms appeared. They construct a globally smooth parameterization associated with the decimation $^{6}$ or chartification ${ }^{7}$ principles. The smoothness of the parameterization is obtained not only within each coarser triangle, but also across patch boundaries and corners. It is also directly related to how well the mesh can be compressed, together with providing good approximations and no degenerated mesh elements.

The application of the MR analysis on the meshes, resulting from these latter algorithms may be based on various subdivision schemes. Most of the existing methods ${ }^{1,3,8}$ benefited from interpolating subdivision filters to provide numerical stability of the fitting operation. But other authors ${ }^{9,10}$ recently proposed a wavelet construction based on the Loop subdivision and the lifting scheme. They managed to overcome the Khodakovsky ${ }^{11}$ filter drawbacks by constructing stable schemes for the wavelet analysis and synthesis with a linear time complexity. These actual state of the art mesh compression methods, presented before, generally apply a global wavelet decomposition, using the same filters on the entire surface. The produced coefficient amplitude and polar angle are hence heterogeneously distributed. They mainly depend on the remeshing algorithm and the prediction power of the considered scaling functions.

\section{Contributions:}

The previous diagnostic encouraged us to propose a new MR analysis framework designed to improve the compression bitrates and produce a more flexible 3D visualization, adapted to user resources and expectations. This framework uses a segmentation algorithm based on the wavelet coefficient magnitude, which every remeshing or compressing algorithm tends to minimize. Each produced partition share surface roughness homogeneity, inherently reflected by wavelet coefficient magnitude. They are ideally suited for adapting the decomposition, quantization and/or encoding steps, in order to reduce the information to encode or set up view-dependent refinements. Our main contribution is the use of our adaptive framework to propose a ROI decoding based on the user's point of view. The results we obtained, when considering view-dependent reconstructions of the "Horse" model, showed significant bitrate improvements compared to view-independent approaches.

\section{OVERVIEW OF OUR APPROACH}

The main features of our framework are presented in Fig. 1 and detailed in the following sections.

The input of the analysis is a semi-regular mesh $M_{s r}$ obtained by applying a remeshing algorithm on an original irregular mesh $M_{i r}$. A global wavelet analysis first decomposes this SR model into $n$ coarser meshes $M_{1}, M_{2}, \ldots, M_{n}$. Then, a shape segmentation method, ${ }^{12}$ presented in Section 3, processes the wavelet coefficient magnitude in all resolution levels. Any particular mesh $M_{i}$ of this hierarchy can be the "support" for the classification and segmentation steps. We compared the application of these last-mentioned steps on the first 


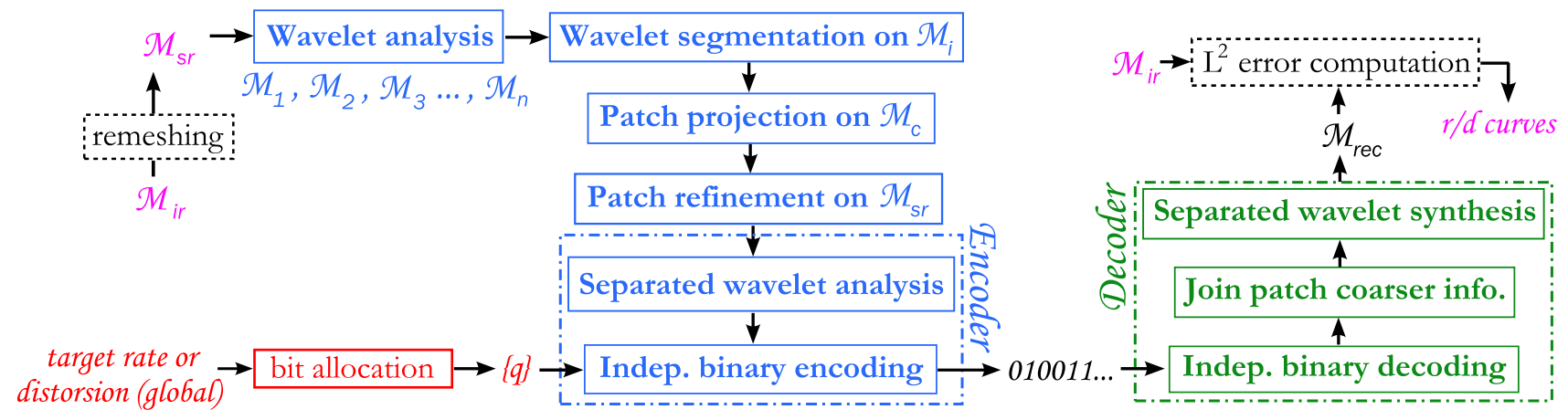

Figure 1. Main features and stages of our framework which aims at locally encoding a semi-regular mesh $M_{s r}$ (remesh of the original irregular model $M_{i r}$ ). This allows a robust and efficient reconstruction $M_{r e c}$ on the client side.

and last $\left(M_{c}\right)$ decomposition levels. Hence it is easy to compute a global mean measure which takes into account the entire hierarchy on which each coefficient reveals the high frequencies lost during all the coarsifications applied from the original model. The goal of this segmentation is to construct homogeneous regions according to the coefficient magnitude, that every remeshing or compressing algorithm tends to minimize.

Once we have partitioned the first resolution level, we project it on the coarsest resolution level $M_{c}$, to provide thereafter, the possibility to compress independently each constructed partition. We chose a projection rather than a patch remeshing, to preserve the initial remeshing properties. Then a patch refinement is necessary for both considered rules, to conclude our hierarchical partitioning. These stages are described in Section 4 together with the locally adapted MR analysis we used. Finally our experimental results are presented in Section 5, showing the additive information needed for proposing a patch independent coding. Other possible applications (which can benefit from the described redundancy) are finally illustrated.

On the synthesis side, after an independent entropy and zerotree decoding, the coarsest patches are first glued and followed by a reversed wavelet transform. The reconstructed object $M_{r e c}$ can finally be compared to the original one, so as to evaluate the distortion.

\section{SHAPE PARTITION ALGORITHM}

To obtain regions gathering common characteristics, most of the existing segmentation algorithms are based on the planarity information of the faces ${ }^{13,14}$ or the discrete surface curvature computed in each vertex. ${ }^{15,16}$ The watershed algorithm used by these latter approaches tend to extract only regions surrounded by high curvatures and do not handle correctly the boundaries between patches, which are either fuzzy or jagged. The method developed by Lavoué et al. ${ }^{17}$ overcomes these drawbacks thanks to a K-Mean classification algorithm instead of the watershed, together with boundary rectifications, in order to more precisely detect curvature transitions on CAD objects.

We adapted their algorithm, formerly conceived for the compression of CAD objects, with sharp edges and corners that generally separate smooth regions. Our extension uses the same concepts based on the production of homogeneous partitions. However we consider the wavelet coefficient magnitude instead of the principal curvature values.

\subsection{Wavelet coefficients as a segmentation criterion}

The decorrelation power of the wavelets has been demonstrated for images, by its incorporation in the JPEG2000 standard, and even for meshes in the MPEG4 standard. We propose to study the wavelet coefficient magnitude distribution associated to a global MR analysis on Normal Meshes. ${ }^{5}$ But the input model can also be remeshed by any algorithm, regardless of its wavelet distribution characteristics.

The non-lifted butterfly wavelet transform used for this task belong to the class of second-generation wavelets introduced by Sweldens, ${ }^{18}$ specifically designed to adapt to irregular point sets. For the usual 3D models, 


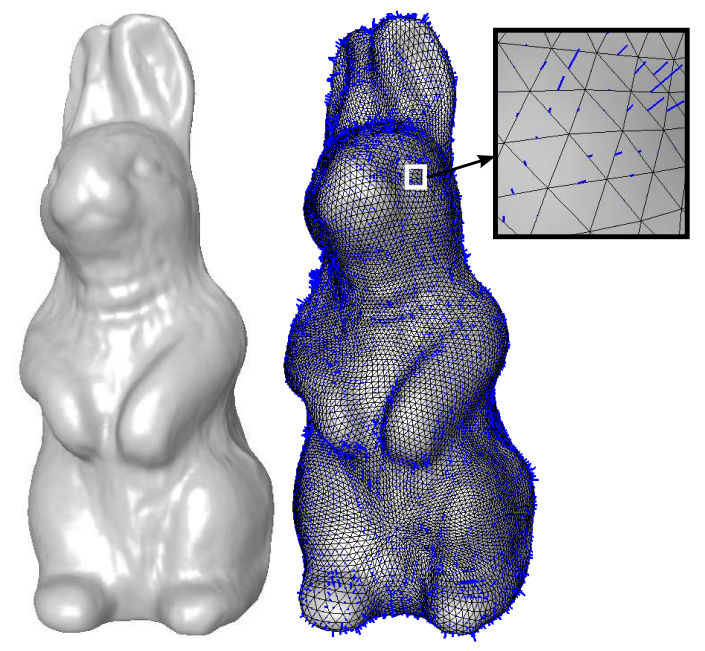

Figure 2. Left: Original Normal "Rabbit"; Right: Wavelet coefficients represented as 3D vectors linked to edges of the $1^{\text {st }}$ resolution level (multiplication factor: 20 ).

subdivision surfaces are a well adapted prediction in homogeneously smooth regions, because they generate a smooth finite surface. Consequently the highest coefficient magnitudes are associated to the high frequencies (features, textures or noise) of the model. A segmentation based on their polar angle can also be considered if the SR model results from a different kind of remeshing algorithm, like MAPS. ${ }^{4}$ However the distribution histogram produced by a Normal Mesh (presented in the last picture of Fig. 3) is too narrow around zero for applying this kind of classification, even after a Gaussian Normalization (used in our results).

For 3D meshes, the facet refinement during the MR synthesis, consists in first applying a canonical quadrisection. For each triangle, the position of the three newly added vertices is obtained by the prediction operation, followed by the wavelet coefficient addition. Consequently wavelets are commonly represented in association with their corresponding coarser edges, as we can see in Fig. 2. The classification and segmentation algorithms we adapted were formerly designed to partition mesh data associated to vertices. We tested different scenarii considering directly the edge-associated information or the data means computed on incident vertices or facets. The vertex-based classification produced the best results.

We indicate in Fig. 3 several coefficient distributions on the "Venus" model. A Gaussian normalization was used, to trim extreme values of the interval, leading to even better results. This confidence interval creation is possible because the associated histograms (presented in Fig. 3) have a Gaussian normal distribution. But as the segmentation algorithm can only be applied on one mesh of the hierarchy, we propose to aggregate all the coefficients in one mesh so as to take into account all the high frequencies lost during the coarsification produced by the subdivision context. Picture $(f)$ of Fig. 3 illustrates this kind of aggregation, on the first resolution level. We compare it with our second aggregation rule in next subsection.

On globally smooth models, the coefficient magnitude distribution allows to identify high curvatures characterizing the eyes, ears, feet or the nose of the "Rabbit" and "Horse" models. This distribution emphasizes also the textured parts, such as the hair of the "Venus" head. For them, another prediction scheme, decomposition or quantization can provide better compression results.

\subsection{Mesh classification and segmentation stages}

Our adaptation of the classification algorithm of Lavoué et al. ${ }^{17}$ was first used to create two groups of vertices, one with the smallest amplitudes and the other with the highest ones. We indicate the results obtained for the coefficient magnitude, in our context, but it is also possible to combine other criteria (for example the polar angle, for some kinds of remeshing). 


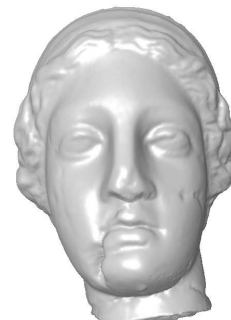

(a)

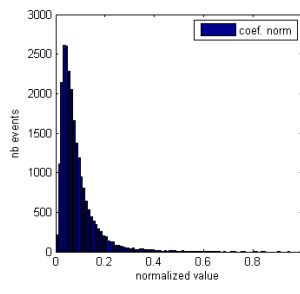

(b)

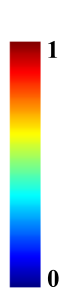

(c)

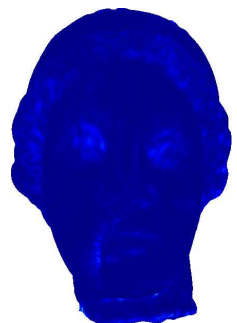

(d)

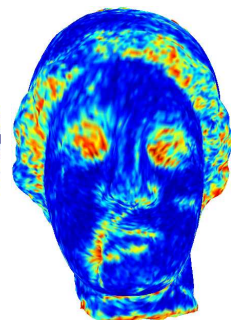

(e)

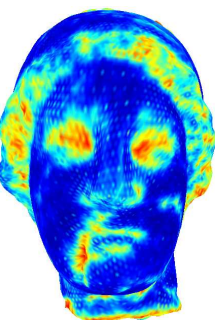

(f)

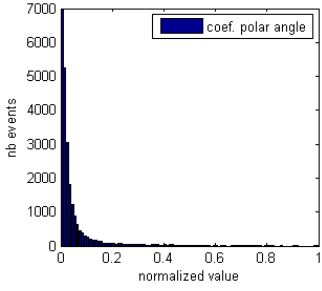

(g)

Figure 3. Distribution of wavelet coefficient magnitudes on the Normal "Venus". The decomposition was produced with the non lifted Butterfly scheme. (a) Original Normal "Venus" model; (b) Histograms of the normalized coefficient magnitude for all resolution levels; (c) Color scale used for the next figures; (d) Distribution of the normalized coefficient magnitude on $1^{\text {st }}$ decomposition level; (e) Same distribution but using a Gaussian normalization; (f) Distribution means of all the Gaussian normalized magnitudes grouped on the $1^{\text {st }}$ level; (g) Histograms of the normalized polar angle for all resolution levels.

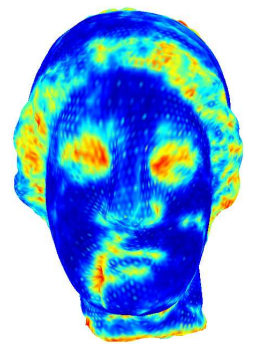

(a)

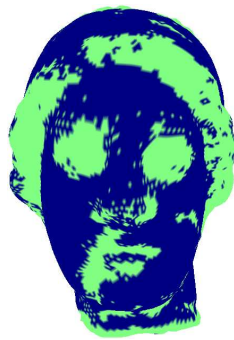

(b)

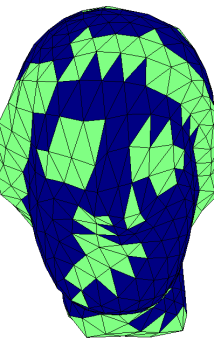

(c)

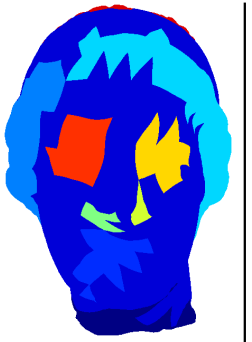

(d)

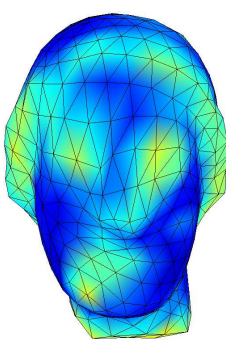

(e)

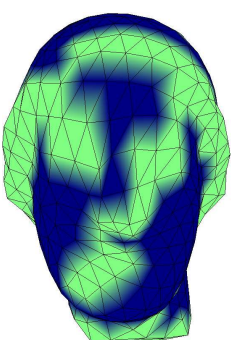

(f)

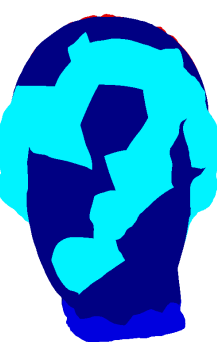

(g)

Figure 4. Classifications and segmentations based on the coefficient magnitude for our two different rules, on Normal "Venus". (a, e) Distribution mean of all Gaussian normalized coefficient magnitudes grouped on the $1^{\text {st }}$ and last level; (b, f) Two-clustered classification on the $1^{\text {st }}$ and last level; (c) Classification projection on the coarsest level; (d, g) Resulting segmentation on the finest mesh.

The construction of the partitions, using the region growing algorithm, consists in transmitting the studied measure from vertices to triangles, starting from seed triangles having their three vertices on the same cluster. In comparison to Lavoué algorithm, we didn't use the region merging step, aiming at reducing the oversegmentation, because our coarse projection associated to the first aggregation rule or the coarse classification produced by our second rule already play this role.

Thanks to this framework, a mesh decomposition in a finite number of regions can be created at a given resolution level, as we can see in Fig. 4 and 5 for the "Venus" and "Horse" models. The colors used for the partitions were randomly chosen. For every classification picture, the dark color is always associated to the smooth cluster. Since our goal consists in an independent analysis and synthesis adapted to the specificity of each patch or the user's point of view, we need to apply a separate MR analysis or synthesis in each produced partition. We describe, in the next section, the method used and comment on the rest of the pictures.

\section{LOCAL MULTIRESOLUTION ANALYSIS COMPUTED ON PRODUCED PARTITIONS}

In order to analyze, quantify, and encode separately each connex region, we need to decompose independently each one into several levels. That's why we considered two different strategies in association with our two aggregation rules. We obtained good approximations on the coarsest level, in comparison with the former segmentation linked to the first resolution level. The final stage projects back the approximations on the finest model (the original one), to begin the local analysis. 


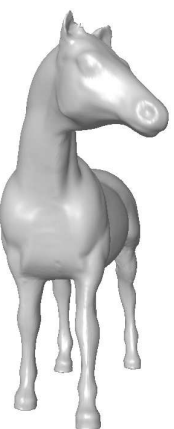

(a)

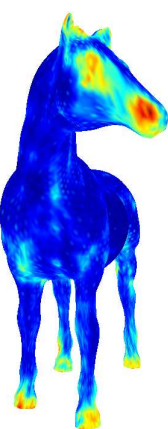

(b)

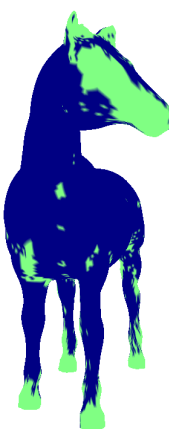

(c)

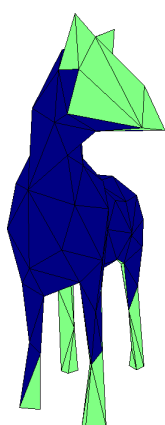

(d)

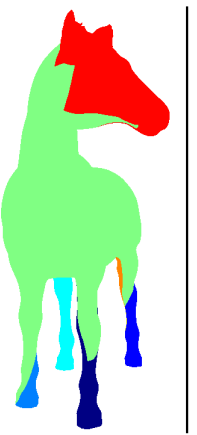

(e)

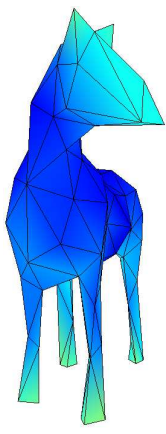

(f)

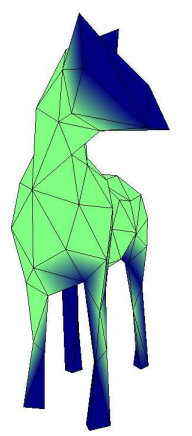

(g)

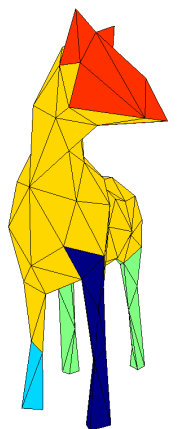

(h)

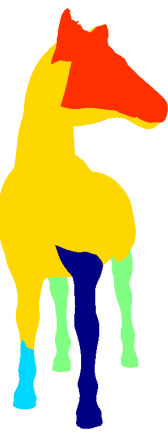

(i)

Figure 5. Classifications and segmentations based on the coefficient magnitude for our two different rules, on Normal "Horse". (a) Original Normal "Horse" model; (b, f) Distribution mean of all Gaussian normalized coefficient magnitudes grouped on the $1^{\text {st }}$ and last level; (c, g) Two-clustered classification on the $1^{\text {st }}$ and last level; (d) Classification projection on the coarsest level; (h) Partitions obtained after the region growing step; (e, i) Final regions projected on the finest mesh.

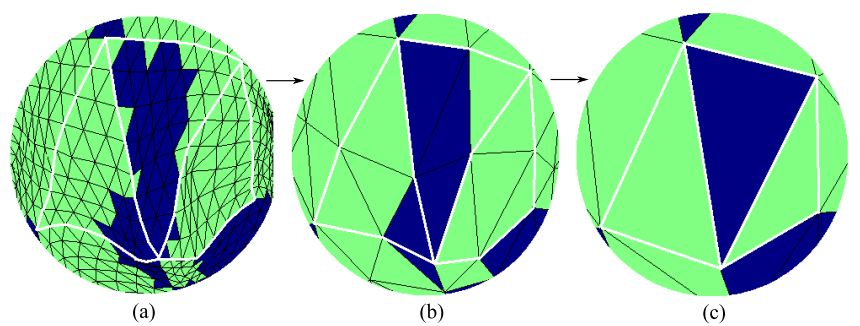

Figure 6. Example of the coarse facet cluster affiliation determination, after two successive coarsifications with our projection rule, used in association with our first aggregation rule. The smooth cluster is represented in dark color.

\subsection{Projection of the segmentation in the hierarchy}

We now detail the projection of our first considered classification (based on the first aggregation rule), on the coarser resolution levels. If we assume that the segmentation was computed on the resolution level $n$, the projection will start on the immediately coarser level $(n+1)$ and will continue until the coarsest level or until the produced regions are too far away from the initial ones.

The coarse triangle cluster affiliation is determined according to its incident four finer facets. In the rule we adopted (illustrated in Fig. 6), if at least three of the four fine triangles belong to a given cluster, the corresponding coarser triangle will also belong to this cluster. If an equality occurs, we favor the non smooth cluster (light color in Fig. 6) to ensure no feature is left out in the non-smooth cluster.

\subsection{Independent decompositions}

Once the projection on the finest level is completed, the mesh can be locally analyzed, considering for example two different prediction schemes according to the cluster affiliation of each patch, to separate treatments on smooth parts from rough sites. But we can also think of a patch-based specific and different treatment, depending on its high frequency level. In any cases, it produces as many "wavelet files" as created regions.

\subsection{Zerotree and entropy coding}

We have considered to encode the wavelet coefficients with the Khodakovsky et al. ${ }^{11}$ zerotree representation, which is based on the coefficient distribution in the MR analysis hierarchy. Their code uses the parent-child coefficient correlations, minimizing the significant bits to encode at each step. Moreover the highest order bits 
Table 1. Characteristics of our two segmentation rules on three usual models remeshed by two different algorithms. '\# vtx' = number of vertices and '\# face' = number of faces on SR models. '\# reg. 1/2' = number of regions obtained with the first/second aggregation rule. '\% rough' = rough triangle rate on the underlying classification.

\begin{tabular}{|c||c|c||c|c|c||c|}
\hline & \# vtx & \# face & \# reg. 1 & \% rough & \# reg. 2 & \% rough \\
\hline Venus Normal & 163,842 & 327,680 & 12 & $30 \%$ & 5 & $38 \%$ \\
\hline Venus MAPS & 198,658 & 3297,312 & 16 & $47 \%$ & 7 & $43 \%$ \\
\hline Horse Normal & 112,642 & 225,280 & 6 & $27 \%$ & 5 & $36 \%$ \\
\hline Feline Normal & 258,046 & 516,096 & 11 & $33 \%$ & 9 & $27 \%$ \\
\hline
\end{tabular}

of the largest magnitude coefficients are sent first. This strategy allows to obtain, for each bitrate, the best reconstructed model that produces the smallest distortion. A scalar quantization is generally used, so there are indeed three independent coders (one for each coordinate). Finally, an arithmetic coding is added to further compress the data.

Apart from the wavelets, the compression file size also includes the scale coefficients corresponding to the encoding of the coarsest mesh. We used the embedded coarsest geometry encoding method of Khodakovsky et $a l .{ }^{11}$ where the coarsest geometry is stored with the zerotree representation. The connectivity is compressed with a single rate (Touma and Gotsman ${ }^{19}$ ) coder.

The data flow are now ready to be transmitted over the network and reconstructed on the client side. All the analysis stages we described need to be reversed on the decoder side. We now present the possible applications of our framework.

\section{EXPERIMENTAL RESULTS AND APPLICATIONS}

\subsection{Compression results}

Fig. 7 exhibits three rate/distortion (r/d) curves produced with the global MR analysis (blue) and our local extension with its two aggregation rules (green and red), for three studied models. The non lifted butterfly wavelets were used everywhere for both global and local cases. The remeshing error indicated in this figure, corresponds to the distortion produced by the underlying SR remeshing. This limit represent the highest quality the considered encoders can reach.

$P S N R=20 \log _{10}$ peak/d where peak is the bounding box diagonal and $d$ the $L^{2}$ relative error, corresponding to the following $L^{2}$ distance $d(X, Y)$ between the surfaces $\mathrm{X}$ and $\mathrm{Y}$ :

$$
d(X, Y)=\left(\frac{1}{\operatorname{area}(X)} \int_{x \in X} d(x, T)^{2} d x\right)^{\frac{1}{2}}
$$

This distance was computed with the MESH tool ${ }^{20}$ by taking the max of $d(X, Y)$ and $d(Y, X)$. The rate is reported in bits per vertex $(\mathrm{b} / \mathrm{v})$ according to the number of vertices in the original irregular input mesh. We can see the amount of extra information necessary for the local analysis compared to the global one, using a common treatment for all the regions of the surface.

The associated number of created regions and the percentage of the identified clusters (which can be smooth or rough) are reported in Table 1, for a better understanding of the curves. This table also compares the segmentations produced by both aggregation rules. First of all, we can see that the second rule has a tendency to produce a less amount of regions, which generally denotes fewer boundary edges. This observation is interesting for compression purposes, for which the number of additional information produced by our independent coding will be smaller. However the clusters produced by this second rule are visually less accurate when we compare them to the finest classification displayed at the beggining of each previous figure. 

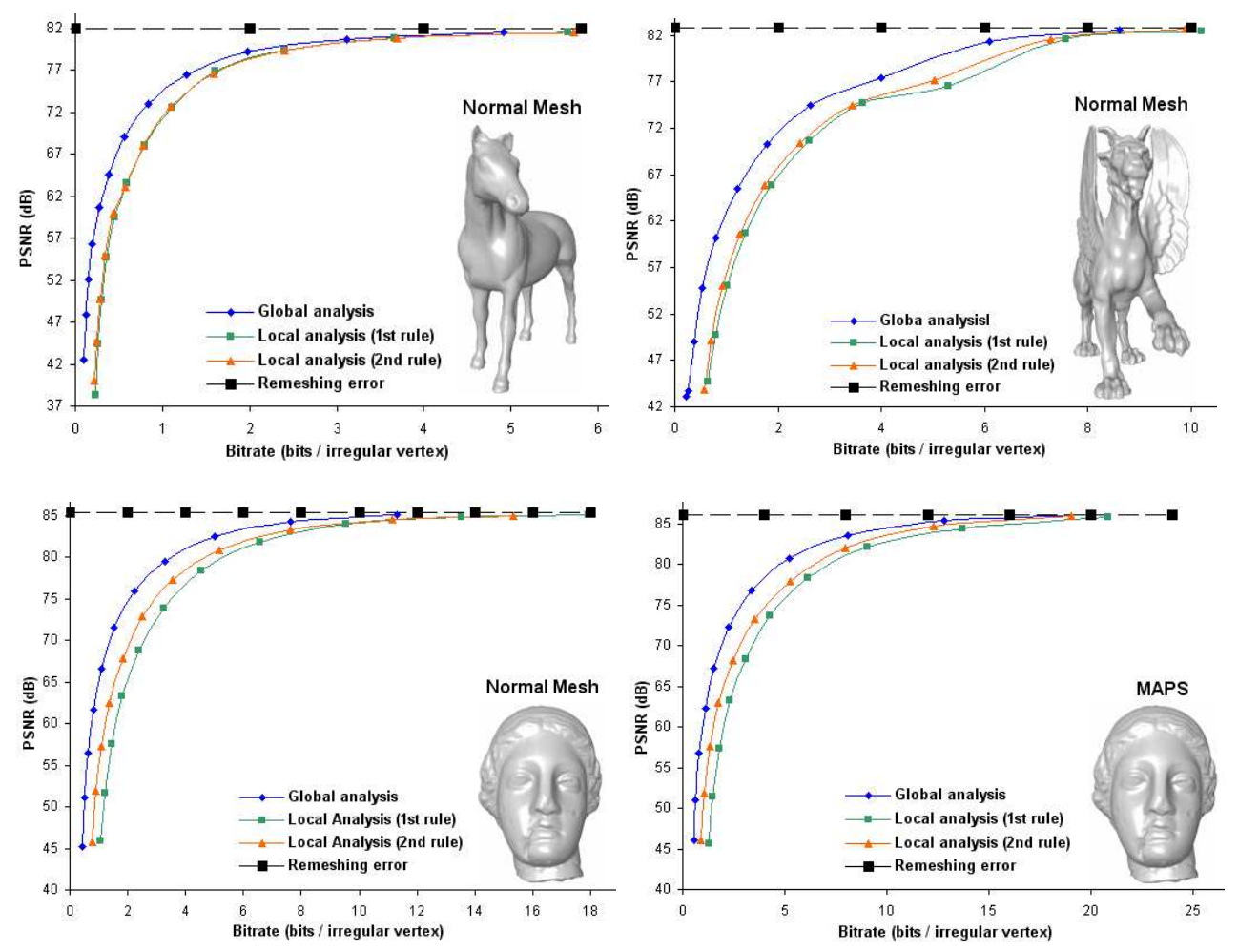

Figure 7. Rate-distortion curves for the studied 3D models remeshed by the Normal Mesh or MAPS algorithms. We considered the global MR analysis (blue curves) and our local extension with its two aggregation rules (green and red curves). The non lifted butterfly wavelets were used everywhere for both global and local cases.

The extra cost of our local framework when applying the same treatment to each partition can be evaluated to 3 or $4 \mathrm{~dB}$ in average compared to the global treatment, for a bitrate greater than $0.5 \mathrm{~b} / \mathrm{v}$. The redundancy introduced by the partitioning can be compensated by a well adapted decomposition on each region, that can counterbalance the redundant information added by our local analysis. For smooth clusters, the prediction produced by the Butterfly subdivision scheme appears really accurate because the wavelet coefficients are close to zero. For other regions, higher details are generally needed to represent high frequencies, so another type of prediction can be a benefit to counterbalance our redundancy problems.

Apart from compression, our framework can also serve as a basis for lots of other applications. We present in the next section two examples, when considering selective refinement depending on the user's resolution terminal or point of view. Subdividing smooth partitions can be sometimes sufficient when visualizing the object in a low resolution device. Finally, if the user is interested in visualizing only a given mesh region, the coarsest resolution level can be sufficient for other partitions.

\subsection{Possible selective refinements}

Nowadays it is common for interactive applications to exchange 3D objects via Internet, between a server and many clients. For these cases, the transmission, decoding and rendering steps need to be fast and efficient. Since the 3D models are more and more densely sampled, a view-dependent streaming can accelerate their processing and rendering, where object regions can be more refined than others, depending on the user's point of view.

Lots of selective simplification or refinement methods exist to adapt vision parameters in real-time. The authors first considered terrain modeling, parametric surfaces (B-splines or NURBS), and then irregular ${ }^{21}$ and semi-regular ${ }^{22-24}$ meshes. We focus on the ones which use a compression method based on a prior surface partitioning, to propose a selective reconstruction in the client side. ${ }^{2-24}$ Hence the server can partition the 


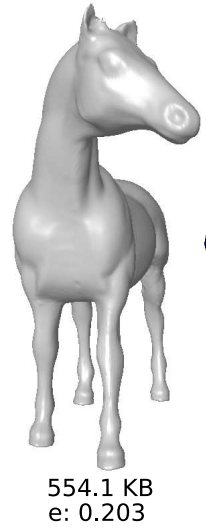

(a)

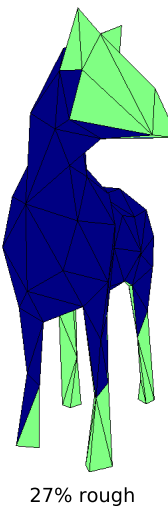

(b)

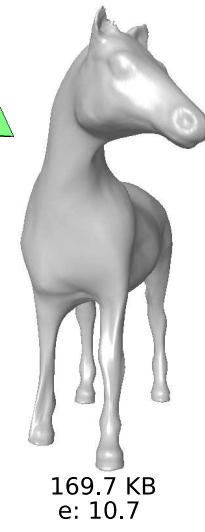

(c)

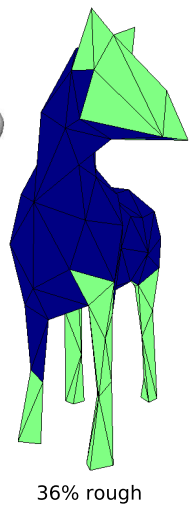

(d)

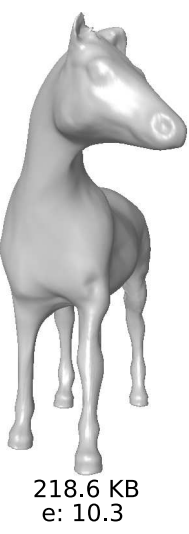

(e)

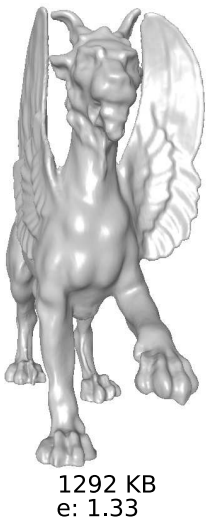

(f)

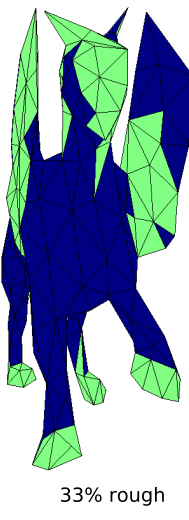

(g)

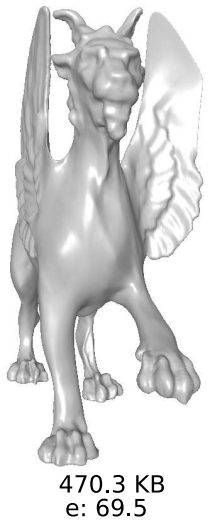

(h)

Figure 8. Possible reconstructions with our flexible local framework on the "Horse" and "Feline" models. (a, f) Reconstruction obtained from a global wavelet decomposition. (b, g) Two-clustered classifications using the first aggregation rule and projected on the coarsest level; (d) same classification but based on the second rule for the "Horse" model. (c, e) Reconstructions produced with the non lifted Butterfly subdivision, followed by the wavelet addition in the non smooth parts. We have reported the corresponding coding file size in bytes and $L^{2}$ error in units of $10^{-4}$ for each considered reconstruction.

object and send each region individually or sequentially to the client, in order to optimize their resolution according to their visibility. These latter methods are generally robuster to transmission errors than global approaches.

Such as the work recently published by Cheng et al. ${ }^{24}$ we propose a part-based mesh reconstruction with different focuses depending on the user's expectations. Our method can decode perfectly the full details of a meaningful part, without doing it for other patches. Moreover we obtain a better rendering for the identified "non meaningfull" regions using the subdivision surfaces, instead of keeping the coarse facets. We illustrate this improvement in Fig. 8, which also demonstrate that for the identified smooth patches, the wavelets are generally not needed for objects visualized on low resolution screens. It can hence accelerate the rendering with a comparable visual quality. For each reconstruction, illustrated in Fig. 8, we specified the corresponding compressed file size in Kbytes and the $L^{2}$ error in units of $10^{-4}$ (Hausdorff distance). Pictures $(a)$ and $(f)$ present the reconstructions obtained when applying a global synthesis for comparison.

The first observation is that the single subdivision applied on the smooth regions produces good reconstructions with a lower $L^{2}$ error than without any subdivision. More specifically, with approximately twice fewer bytes, we obtain a good reconstruction of the "Horse" model, which preserves the important characteristics of the object. This decompression can be sufficient for a visualization on a low resolution device. The "Feline" model is also well reconstructed with our first local rule, but the file size reduction is less significant because it contains a more features and textured parts.

Regarding a given Hausdorff distance $\left(e_{1}\right)$, we finally compared the visual quality of two different reconstructions, considering our local approach (used to produce picture (e) of Fig. 8), and the global synthesis. The results, illustrated in Fig. 9, highlight that for the same Hausdorff distortion, our reconstruction better preserves the features (feet and head), for which a more important number of bits were allocated than for the body. "Horse" head was zoomed in, to better appreciate it. This observation is highlighted by the second distortion measure we have considered $\left(e_{2}\right)$. This objective structural distortion measure (called MSDM) was introduced by Lavoue et $\mathrm{al}^{25}$ in 2006. MSDM uses geometric metrics, based on curvature analysis computed on local windows in the analysed mesh. The objects are hence compared and evaluated through a subjective experiment, based on human evaluation of a set of distorted objects. Similar objects have a distortion close to 0 . 

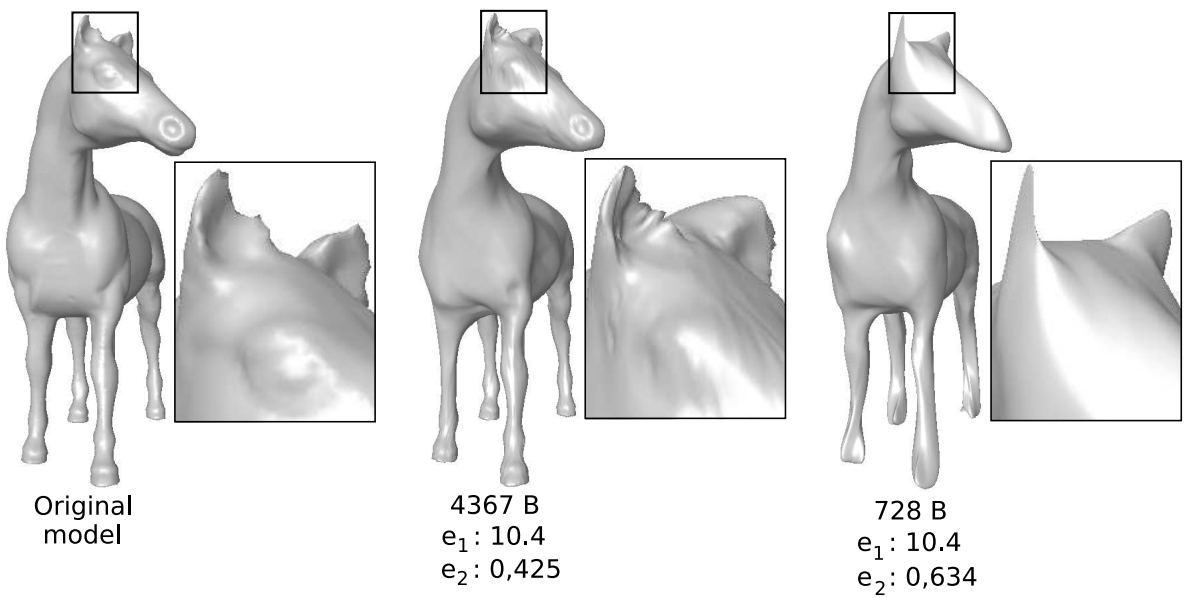

Figure 9. "Horse" model reconstructions based on local and global considerations, with the same reconstruction error. Left picture remembers the original irregular mesh. The reconstruction presented in the middle, was produced with our local framework where subdivision is only followed by wavelet addition in non-smooth regions. Right reconstruction was obtained from a global wavelet decomposition. We have reported the corresponding coding file size in bytes (B). The distortion errors $e_{1}$ and $e_{2}$ correspond respectively to Hausdorff distance (in units of $10^{-4}$ ) and Lavoué et al. ${ }^{25}$ MSDM metric.

\subsection{ROI decoding}

We also considered applications where the user is interested in a caracteristic part of the mesh, as for example "Horse" head. In this context, we compared in Fig. 10 two reconstructions of the "Horse" model, with similar local distortion computed in "Horse" head (with Hausdorff distance). We also compared the compressed file sizes, associated to view-independent method (global: on top of the figure) and our view-dependent approach (down), for which the underlying segmentation is mentioned. In this latter case, our method is able to transfer and decode only details associated to the considered region. The other wavelet coefficients can be sent afterwards, when the user's point of view changes.

Hence, for a similar visual quality (when user's interest only focuses on the head region), our approach saves more than $50 \%$ of the bitrates. Sim et al. ${ }^{23}$ obtained comparable results with their optimized bit allocation which takes into account only visible disjoint fragments in the mesh. Since our mesh partitioning isolates features which generally correspond to semantic object parts, the user's ROI selection can be quicker with our method, because the actual ROI we can consider are tightly linked to our underlying segmentation. Hence, instead of indicating a set of facets or fragments corresponding to the desired ROI, the user just need to select a partition produced by our prior segmentation. In this context, our first partitioning rule gives clearly better results, except for "Horse" model for which our two segmentations are very close.

\section{CONCLUSION AND FUTURE WORK}

The main contribution of this work is the use of our patch-based decomposition framework, to propose a viewdependent visualization, adapted to user's resources and expectations. This framework was formerly conceived to apply different decompositions, quantizations and coding treatments on specific identified regions. It provides an entire adaptive compression chain, based on any prior segmentation of the mesh surface. Our algorithm is fast and applicable to any semi-regular triangulated surface. We showed that the redundancy introduced by the local MR analysis can be compensated by a well adapted treatment on each analyzed region.

We first propose, as a future work, to improve the prediction schemes in identified non smooth regions (where actual subdivision schemes don't propose good predictions). It can hence reduce the wavelet coefficients, and improve the compression bitrates on natural objects. We can think to account for the anisotropy of the surface causing these high coefficients. Moreover, fractal analysis could also be considered in noisy regions. 


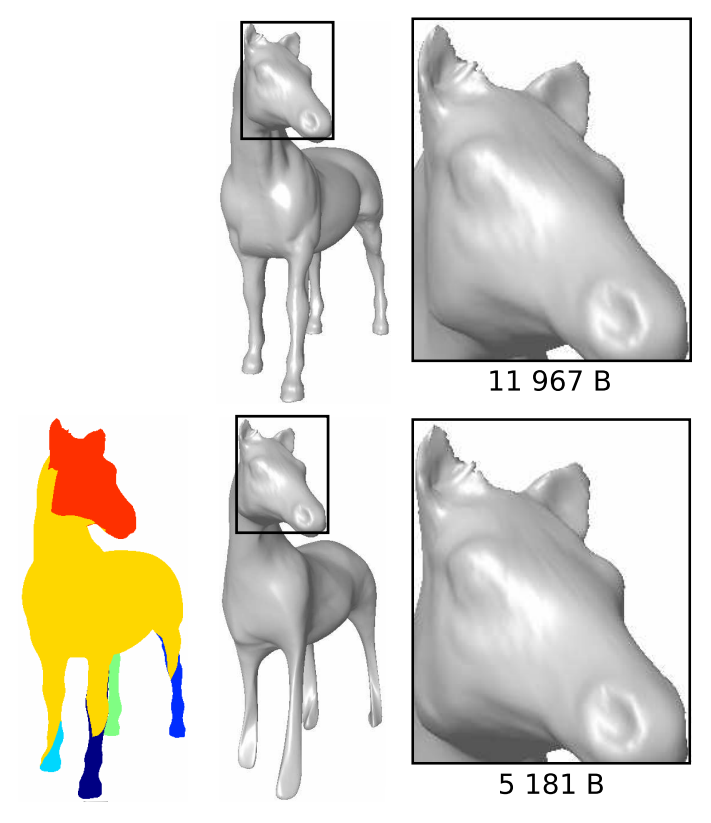

Figure 10. Compressed file size comparison for "Horse" model analysed with global method (top) and our adaptive approach (down). For both reconstructions, local distortion computed in "Horse" head is equivalent.

We also would like to improve the rate/distortion (r/d) results produced by our local wavelet analysis. Following the work done by Payan et $a l .{ }^{26}$ and Sim et al. ${ }^{23}$ we would like to further optimize the wavelet quantization and bit allocation for each produced patch, with respect to its distortion contribution to the entire surface. The principle is to use a Lagrangian optimization in each subband of each region.

Other applications could benefit from our partitioned analysis, like watermarking. It would allow to apply different marks according to the visual aspect of the surface. Another interesting point is that partition coding includes extra information that improves the reliability of data transmission in telecommunication channels. So another possible application would be error-resilient 3D mesh coding.

\section{REFERENCES}

1. M. Lounsbery, T. D. DeRose, and J. Warren, "Multiresolution analysis for surfaces of arbitrary topological type," ACM Transactions on Graphics 16(1), pp. 34-73, 1997.

2. M. Eck, T. DeRose, T. Duchamp, H. Hoppe, M. Lounsbery, and W. Stuetzle, "Multiresolution analysis of arbitrary meshes," in SIGGRAPH '95: Proceedings of the 22nd annual conference on Computer graphics and interactive techniques, pp. 173-182, (New York, NY, USA), 1995.

3. P. Gioia, "Reducing the number of wavelet coefficients by geometric partitioning," Comput. Geom. Theory Appl. 14(1-3), pp. 25-48, 1999.

4. A. W. F. Lee, W. Sweldens, P. Schröder, L. Cowsar, and D. Dobkin, "Maps: Multiresolution adaptive parameterization of surfaces," Computer Graphics 32(Annual Conference Series), pp. 95-104, 1998.

5. I. Guskov, K. Vidimce, W. Sweldens, and P. Schröder, "Normal meshes," in Siggraph 2000, Computer Graphics Proceedings, pp. 95-102, 2000.

6. A. Khodakovsky, N. Litke, and P. Schröder, "Globally smooth parameterizations with low distortion," $A C M$ Trans. Graph. 22(3), pp. 350-357, 2003.

7. I. Guskov, "Manifold-based approach to semi-regular remeshing," Graph. Models 69(1), pp. 1-18, 2007.

8. A. Khodakovsky and I. Guskov, "Compression of normal meshes," in Geometric Modeling for Scientific Visualization. Springer-Verlag, 2003.

9. M. Bertram, "Biorthogonal loop-subdivision wavelets," Computing 72(1-2), pp. 29-39, 2004. 
10. D. Li, K. Qin, and H. Sun, "Unlifted loop subdivision wavelets," in PG '04: Proceedings of the Computer Graphics and Applications, 12th Pacific Conference on (PG'04), pp. 25-33, IEEE Computer Society, (Washington, DC, USA), 2004.

11. A. Khodakovsky, P. Schröder, and W. Sweldens, "Progressive geometry compression," in Siggraph 2000, Computer Graphics Proceedings, pp. 271-278, 2000.

12. C. Roudet, F. Dupont, and A. Baskurt, "Multiresolution mesh segmentation based on surface roughness and wavelet analysis," Visual Communications and Image Processing 2007 6508(1), p. 65082E, SPIE, 2007.

13. M. Garland, A. Willmott, and P. S. Heckbert, "Hierarchical face clustering on polygonal surfaces," in SI3D '01: Proceedings of the 2001 symposium on Interactive 3D graphics, pp. 49-58, ACM Press, (New York, NY, USA), 2001.

14. D. Cohen-Steiner, P. Alliez, and M. Desbrun, "Variational shape approximation," ACM Trans. Graph. 23(3), pp. 905-914, 2004

15. Y. Sun, D. Page, and J. Paik, "Triangle mesh-based edge detection and its application to surface segmentation and adaptive surface smoothing," in IEEE International Conference on Image Processing, 3, pp. 825-28, (Rochester, NY, USA), 2002.

16. A. Razdan and M. Bae, "A hybrid approach to feature segmentation of triangle meshes," Computer-Aided Design 35(9), pp. 783-789, 2003.

17. G. Lavoué, F. Dupont, and A. Baskurt, "Curvature tensor based triangle mesh segmentation with boundary rectification," in Computer Graphics International, pp. 10-17, 2004.

18. W. Sweldens, "The lifting scheme: A new philosophy in biorthogonal wavelet constructions," in Wavelet Applications in Signal and Image Processing III, pp. 68-79, 1995.

19. C. Touma and C. Gotsman, "Triangle mesh compression.," in Graphics Interface, pp. 26-34, 1998.

20. N. Aspert, D. Santa-Cruz, and T. Ebrahimi, "Mesh: Measuring errors between surfaces using the hausdorff distance," in Proceedings of the IEEE International Conference on Multimedia and Expo, I, pp. 705 - 708, 2002. http://mesh.epfl.ch.

21. H. Hoppe, "View-dependent refinement of progressive meshes," in SIGGRAPH '97: Proceedings of the 24th annual conference on Computer graphics and interactive techniques, pp. 189-198, 1997.

22. P. Gioia, O. Aubault, and C. Bouville, "Real-time reconstruction of wavelet-encoded meshes for viewdependent transmission and visualization.," IEEE Trans. Circuits Syst. Video Techn. 14(7), pp. 1009-1020, 2004.

23. J.-Y. Sim, C.-S. Kim, C. Kuo, and S. Lee, "Rate-distortion optimized compression and view-dependent transmission of 3-D normal meshes," 15, pp. 854-868, July 2005.

24. Z.-Q. Cheng, H.-F. Liu, and S.-Y. Jin, "The progressive mesh compression based on meaningful segmentation," Vis. Comput. 23(9), pp. 651-660, 2007.

25. G. Lavou, E. D. Gelasca, F. Dupont, A. Baskurt, and T. Ebrahimi, "Perceptually driven 3d distance metrics with application to watermarking," in SPIE Applications of Digital Image Processing XXIX, (San Diego), Aug. 2006.

26. F. Payan and M. Antonini, "An efficient bit allocation for compressing normal meshes with an error-driven quantization," Comput. Aided Geom. Des. 22(5), pp. 466-486, 2005. 